\title{
THE ORIGIN OF THE EQUINE ENDOMETRIAL CUPS
}

\section{PRODUGTION OF PMSG BY FETAL TROPHOBLAST GELLS}

\author{
W. R. ALLEN AND R. M. MOOR \\ ARC Unit of Reproductive Physiology and Biochemistry, \\ Animal Research Station, 307 Huntingdon Road, Cambridge
}

(Received 16th December 1971, accepted 23rd December 1971)

The gonadotrophin (PMSG) present in the serum of mares between the 40th and 120 th days of pregnancy originates in the uterine endometrial cups. These structures begin to develop on the 36th day of pregnancy opposite a transitory, though well-defined, circumferential thickening of the chorion called the allantochorionic girdle (Ewart, 1897; van Niekerk, 1965; Allen, 1970). The endometrial cups are composed of a discrete and densely packed mass of very large, epithelioid, decidual-like cells. They develop before the allantochorion becomes attached to the endometrium and it has been widely accepted in the past that they are exclusively maternal in origin (Clegg, Boda \& Cole, 1954; Amoroso, 1955). However, recent genetic evidence, derived from the study of interspecific equine hybrids, has indicated that fetal genotype influences the level of PMSG in the maternal blood (Bielanski, Ewy \& Pigoniowa, 1965; Clegg, Cole, Howard \& Pigon, 1962; Allen, 1969a). This led Allen (1970) to suggest that the allantochorionic girdle may play an important rôle in the development of the endometrial cups. We now wish to report on two experiments which provide morphological and biochemical evidence of a new concept of endometrial cup development.

Horse conceptuses were removed at hysterotomy from three mares on the 35 th day of pregnancy. They were immersed in a bath of sterile phosphatebuffered physiological saline $(\mathrm{pH} 7 \cdot 2)$ and the allantochorionic girdle and pieces of other tissues were dissected free. Pieces of allantochorionic girdle, normal allantochorion, fetal skin and maternal endometrium from two mares were then transferred to a culture medium consisting of $20 \%$ fetal calf serum in M199 (Flow Laboratories) containing $50 \mu \mathrm{g}$ insulin, $50 \mu \mathrm{g}$ ascorbic acid, 50 units penicillin and 50 units streptomycin $/ \mathrm{ml}$. The tissues were cultivated in this medium in plastic dishes or bottles (Sterilin) at $37^{\circ} \mathrm{C}$ in $5 \% \mathrm{CO}_{2}$ in air. The medium was changed every 4 days and the concentration of PMSG measured in each sample using the immunoassay described by Allen (1969b). At chosen intervals, cultures were fixed in Bouin's fluid and examined histologically.

In cultures of all four types of tissue, attachment of tissue fragments to the Petri dish or bottle occurred rapidly, and within a few days, large outgrowths of cells were visible around each explant. The most rapidly growing tissue was the fetal skin which reached confluence within 4 days and consisted of elongated fibroblastic cells. The allantochorion grew more slowly and gave rise to sheets 
of squamous, mononucleate, epithelioid cells. The allantochorionic girdle cells exhibited morphological characteristics that were markedly different from those of any of the other cell types; they were large and binucleate and exhibited granular, basiphilic cytoplasm containing numerous small vesicles (Pl. 1, Fig. 1). Moreover, these large binucleate cells were strikingly similar in appearance to the large decidual-like cells that comprise the endometrial cups in the pregnant mare (Pl. 1, Fig. 2).

Gonadotrophin assays of the culture media showed that only the allantochorionic girdle cells produced PMSG; normal allantochorion, endometrium and fetal skin never produced detectable gonadotrophin at any stage. In the allantochorionic girdle cell cultures, PMSG concentrations rose to a maximum of over 400 i.u./ml medium within 30 days of establishment of the cultures

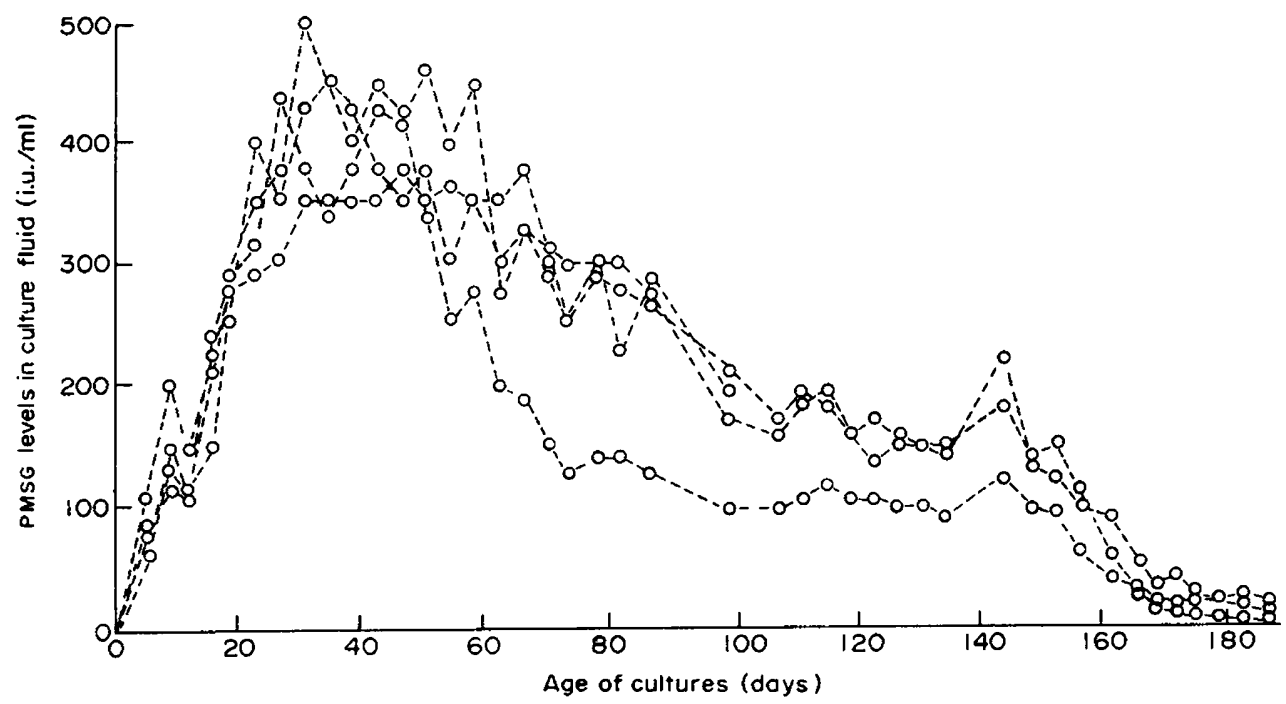

TEXT-FIG. 1. PMSG production by four cultures of horse allantochorionic girdle cells.

(Text-fig. 1). The concentrations remained level for a further 30 days and although they then began to decline, concentrations of 5 to 30 i.u. PMSG $/ \mathrm{ml}$ medium were still present after 180 days of growth. The concentration of PMSG in cultures of allantochorionic girdle cells greatly exceeded even the maximum concentrations of PMSG (100 to $150 \mathrm{i} . \mathrm{u} . / \mathrm{ml}$ ) found in the blood serum

\section{EXPLANATION OF PLATE 1}

FIg. 1. Horse allantochorionic girdle cells cultivated in vitro for 15 days. Note the characteristic multinucleate state of the cells and the accumulation of small vacuoles around the nuclei in most cells. $\times 400$.

Frg. 2. Section of horse endometrial cup at 53 days of pregnancy showing typical deciduallike cup cells, some of which are binucleate. A portion of endometrial gland is visible at the top of the section. $\times 400$.

FIG. 3. Allantochorionic girdle cells 14 days after being grafted beneath the endometrium of a non-pregnant mare. These cells have the same characteristics as endometrial cup cells (see Fig. 2). $\times 400$.

Frg. 4. Allantochorionic girdle cells 14 days after being grafted beneath the tunica albuginea of the testis in a 13-month-old colt. Although the graft site is heavily invaded with leucocytes, the girdle cells are indistinguishable from endometrial cup cells. $\times 400$. 
PLATE 1
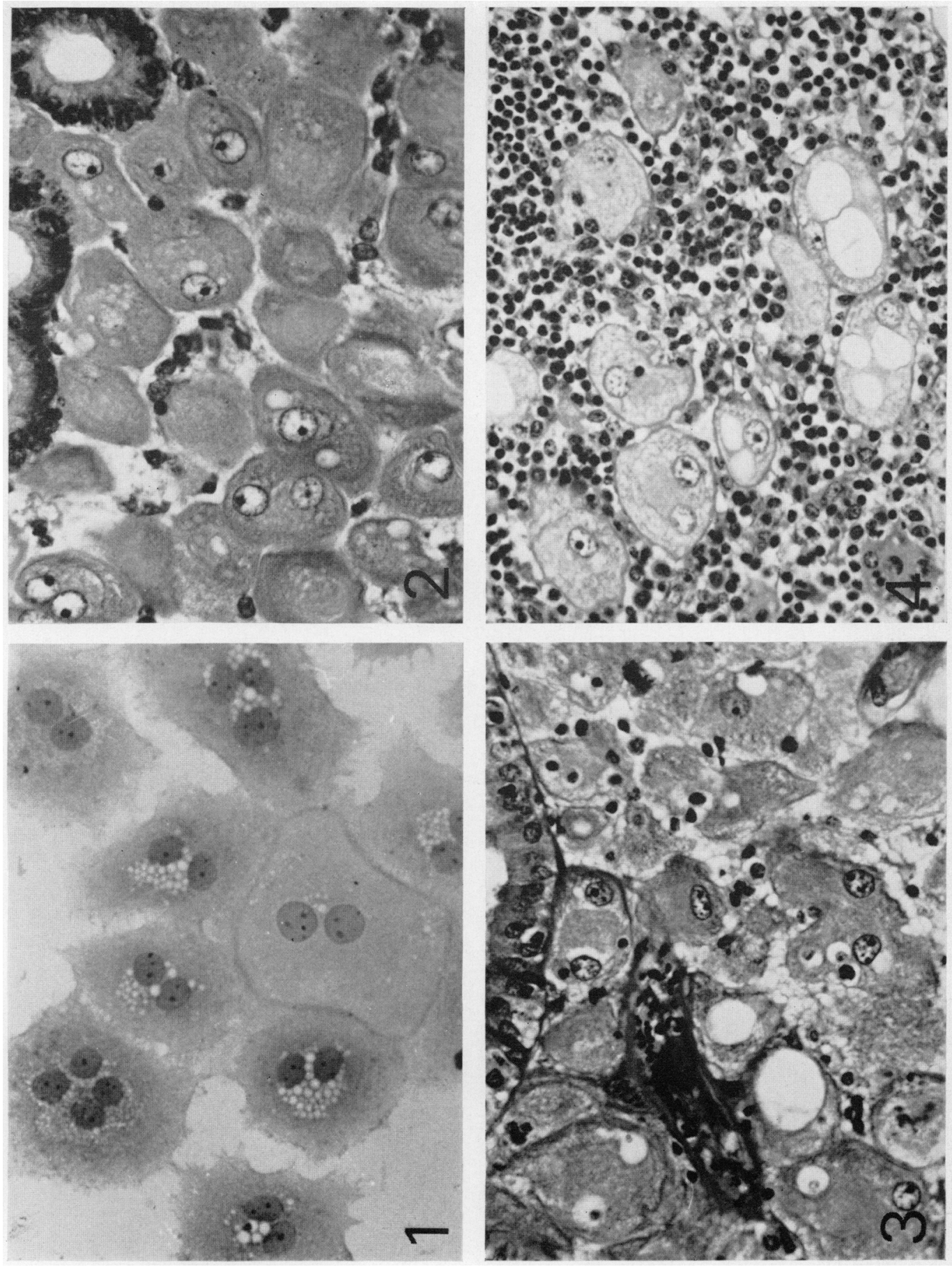

(Facing f) 314) 
of pregnant mares on the 60th day of gestation. Furthermore, PMSG is secreted in vivo for only 80 days while its production in vitro extends over at least 180 days.

The allantochorionic girdle from the third mare was cut into five pieces, each approximately $3 \mathrm{~cm}$ long. Two of these pieces were grafted underneath the endometrium in the non-gravid uterine horn of the same mare from which the conceptus had been removed and the other three were grafted beneath the tunica albuginea of one testis in a yearling colt. Fourteen days later, the graft sites were examined and pieces fixed for histology.

In both graft sites, the allantochorionic girdle cells underwent the same morphological changes as were seen in the cultures in vitro. In the non-gravid uterine horn, three small structures identical in appearance to normal endometrial cups, had developed; these outgrowths were composed of large epithelioid cells indistinguishable from normal endometrial cup cells (Pl. 1, Fig. 3). In the testis, the graft sites were heavily infiltrated with leucocytes, but, nevertheless, small groups of typical large decidual-like cells were still visible (Pl. 1, Fig. 4).

Our experiments have established that specialized equine trophoblast cells recovered at a specific stage of gestation can produce very large quantities of gonadotrophin in vitro for a prolonged period. Only allantochorionic girdle cells have this potential for PMSG synthesis and whether they are grown in vitro or transplanted to other host sites in vivo, these cells exhibit the same distinctive morphological features characteristic of normal endometrial cup cells. These findings strongly suggest that the endometrial cups in the mare are composed of fetal cells, and are not of maternal origin as has been thought previously. This hypothesis is in accord with the original suggestion of Catchpole \& Lyons (1934) that the gonadotrophin found in the mare's blood during early pregnancy is produced by the fetal placenta.

It is noteworthy that allantochorionic girdle cells in culture continue to secrete PMSG for a great deal longer than the endometrial cups in normal pregnancy. This is especially interesting in view of the previous suggestion (Allen, 1970) that the dense accumulation of lymphocytes and plasma cells in the endometrial cups represents a maternal immunologial response to foreign antigens on the endometrial cup cells; the maternal lymphocytic response is expecially marked when the mare is carrying a hybrid fetus. Thus, our present findings give further support to the hypothesis that the life of the endometrial cups in the pregnant mare is normally cut short by maternal immunological defence mechanisms.

This work was supported by the Thoroughbred Breeders Association and the Agricultural Research Council. We wish to thank Mr L. E. A. Rowson, Dr Mary Hay and Professor T. R. R. Mann for their kind help.

\section{REFERENCES}

Allen, W. R. (1969a) Factors influencing pregnant mare serum gonadotrophin production. Nature, Lond. 223, 64.

ALLEN, W. R. (1969b) A quantitative immunological assay for pregnant mare serum gonadotrophin. 7. Endocr. 43, 581 . 
Allen, W. R. (1970) Equine gonadotrophins. Ph.D. thesis, University of Cambridge.

Amoroso, E. G. (1955) Endocrinology of pregnancy. Br. med. Bull. 11, 117.

Bielanski, W., Ewy, Z. \& Pigoniowa, H. (1956) Differences in the level of gonadotrophin in the serum of pregnant mares. IIIrd Int. Congr. Anim. Reprod., Cambridge, 1956, pp. 110-111.

Gatchrole, H. R. \& Lyons, W. R. (1934) The gonad-stimulating hormone of pregnant mares. Am. J. Anat. 55, 167.

ClegG, M. T., BodA, J. M. \& Cole, H. H. (1954) The endometrial cups and allantochorionic pouches in the mare with emphasis on the source of equine gonadotrophin. Endocrinology, 54, 448.

Clegg, M. T., Cole, H. H., Howard, C. B. \& Pigon, H. (1962) The influence of foetal genotype on equine gonadotrophin secretion. F. Endocr. 25, 245.

Ewart, J. C. (1897) A critical period in the development of the horse. Adam \& Charles Black, London.

VAN NIEKERK, C. H. (1965) The early diagnosis of pregnancy, the development of the foetal membranes and nidation in the mare. $\mathcal{F l} S$. Afr. vet. med. Ass. 36, 53. 